\title{
Liquid water and organics in Comets: implications for exobiology
}

\author{
J.T. Wickramasinghe, N.C. Wickramasinghe and M.K. Wallis \\ Centre for Astrobiology, Cardiff University, 2 North Road, CF10 3DY, UK \\ e-mail:ncwick@googlemail.com
}

\begin{abstract}
Liquid water in comets, once considered impossible, now appears to be almost certain. New evidence has come from the discovery of clay minerals in comet Tempel 1, which compliments the indirect evidence in aqueous alteration of carbonaceous chondrites. Infrared spectral indication of clay is confirmed by modelling data in the $8-40 \mu \mathrm{m}$ and $8-12 \mu \mathrm{m}$ wavebands on the basis of mixtures of clays and organics. Radiogenic heating producing liquid water cores in freshly formed comets appears more likely on current evidence for solar system formation. A second possibility investigated here is transient melting in comets in the inner solar system, where thin crusts of asphalt-like material, formed due to solar processing and becoming hot in the daytime, can cause melting of sub-surface icy material a few centimetres deep. Supposing comets were seeded with microbes at the time of their formation from pre-solar material, there would be plenty of time for exponential amplification and evolution within the liquid interior and in the transient ponds or lakes formed as the outer layers are stripped away via sublimation.
\end{abstract}

Received 5 July 2007, accepted 9 August 2007, first published online 17 July 2009

Key words: comets, exobiology, liquid water, panspermia, radioactive heat sources.

\section{Introduction}

Despite the fact that $\mathrm{H}_{2} \mathrm{O}$ was known to make up a large fraction of a comet's composition, liquid water was for long considered impossible. The dominance of Whipple's icy conglomerate model with elementary gases sublimating from frozen material impeded any discussion of a phase transition from ice to liquid water. The first discussions of liquid water in comets can be traced to Hoyle \& Wickramasinghe (1979), who conceived of interior melting due to chemically reactive constituents accreted from interstellar grains, having energy density of around 10 times the latent heat of melting. Radiogenic heat sources had been considered (Whipple \& Stefanik 1966) but long-lived radionuclides were too weak. Then evidence of short-lived ${ }^{26} \mathrm{Al}$ in meteorites led to calculations (Wallis 1980) that if comets formed quickly - within $\sim 1 \mathrm{Myr}$ of a supernova, starting the contraction of the solar nebula - then this would have sufficient energy and abundance to cause interior melting.

A necessary condition for liquid water to be stable within a comet is that the ambient temperature and pressure exceed the corresponding triple point values, $T=273 \mathrm{~K}$ and $p=$ $6 \mathrm{mb}$. These numbers can be relaxed for water containing salts or soluble organics, but we retain them for definiteness. For a static uniform sphere of radius $r$ and density $\rho$ the central pressure is

$p=\frac{2 \pi}{3} G \rho^{2} r^{2}$.
Assuming $\rho \approx 0.3-0.5 \mathrm{~g} \mathrm{~cm}^{-3}$ and setting $p>6 \mathrm{mb}$, Eqn (1) yields

$r>8-20 \mathrm{~km}$

for the minimum radius of a cometary body where gravity sustains a liquid water core. In practice, icy layers and crusts develop significant strength, but if parts of the crust crack and work loose, gas pressure from any liquid bodies are marginally able to blow them off against gravity.

The phase change from ice to liquid water could produce dramatic biological consequences in a comet. Anaerobic or chemotrophic microorganisms present in a freeze-dried dormant state or as spores could commence metabolism and replication. Exothermic reactions involved in metabolism would cause further melting of ices, and gases released in the process would add to the stresses to be supported by an overlying crust.

Evidence for liquid water in comets has been available from interplanetary dust particles and carbonaceous meteorites presumed to originate in comets. Geochemical and textural analyses of minerals in $\mathrm{C} 1$ carbonaceous chondrites provides compelling evidence of aqueous alteration that required liquid water in their parent bodies (McSween 1979), nowadays presumed to be comets (Hoover 2005). Cometary dust (interplanetary dust particles) collected in the atmosphere similarly contains hydrated states, implying aqueous alteration. They include components of clays, serpentines and 
carbonates sharing many mineralogical similarities with chrondritic material (Brownlee 1978).

\section{Primordial melting}

There is compelling evidence from studies of meteorites that the formation of the solar nebula was triggered by the explosion of a supernova. The evidence is contained in extinct radioactive isotopes with short half-lives, principally ${ }^{26} \mathrm{Al}$ and ${ }^{60} \mathrm{Fe}$, and the absence of ${ }^{10} \mathrm{Be}$ produced by cosmic radiation (Zinner 2003).

There is sufficient energy to melt cometary ices in these two short-lived nuclides: ${ }^{26} \mathrm{Al}$ with half-life $0.74 \mathrm{Myr}$ and ${ }^{60} \mathrm{Fe}$ with half-life $1.5 \mathrm{Myr}$. The energy released by the radioactive decay of ${ }^{26} \mathrm{Al}$ to the stable isotope ${ }^{26} \mathrm{Mg}$ is $1.48 \times 10^{13} \mathrm{~J} \mathrm{~kg}^{-1}$. With an interstellar isotope ratio of ${ }^{26} \mathrm{Al} /{ }^{27} \mathrm{Al} \approx 5 \times 10^{-5}$ and the known cosmic abundance ratios of $\mathrm{C}, \mathrm{N}, \mathrm{O} / \mathrm{Al}$ we obtain a ${ }^{26} \mathrm{Al}$ mass fraction in pristine cometary material of $\sim 3 \times 10^{-7}$ (MacPherson et al. 1995; Diehl et al. 1997). The total energy available from radioactive decay of ${ }^{26} \mathrm{Al}$ within a comet is thus $\sim 4.4 \times 10^{6} \mathrm{~J} \mathrm{~kg}^{-1}$. Recently the importance of ${ }^{60} \mathrm{Fe}$ in heating planetesimals has been discussed (Mostefaoui et al. 2004) and similar considerations apply to comets. With a higher relative abundance in cometary material, and a higher energy yield per unit mass, the heat available from the decay of ${ }^{26} \mathrm{Al}$ and ${ }^{60} \mathrm{Fe}$ together could total $3 \times 10^{7} \mathrm{~J} \mathrm{~kg}^{-1}$. This energy yield is two orders of magnitude higher than the heat of fusion of water-ice $\left(3.34 \times 10^{5} \mathrm{~J} \mathrm{~kg}^{-1}\right)$.

The feasibility of primordial liquid water cores is thus firmly established but estimating the duration of a liquid phase requires more detailed calculations. Such calculations involve several poorly defined parameters, including the thermal conductivity of the cometary regolith and the time lapse between the injection of radionuclides into the solar nebula and the formation of comets.

Detailed model calculations of the thermal evolution of radiogenically heated comets have been carried out by Wallis (1980), Yabushita (1993) and Merk \& Prialnik (2003) among others. A limiting factor for heating by short-lived nuclides is the time taken for the cometary bodies to accrete following the injection of radioactive nuclides into the solar nebula. If the process takes much longer than several million years, these radioactive heat sources would have become extinct. Uncertainties still exist in theories of comet formation but a consensus is growing that icy bodies in the inner solar system (Main Belt asteroids) and Edgeworth-Kuiper belt comets formed on timescales less than a few million years. For comets condensing much further out in the solar system over longer timescales, radioactive heating would appear to be weaker, coming from the nuclides ${ }^{232} \mathrm{Th},{ }^{238} \mathrm{U}$ and ${ }^{40} \mathrm{~K}$.

Wallis (1980) considered a heat-conduction model with ${ }^{26} \mathrm{Al}$. Using the thermal diffusion equation, he found that a comet of radius $\sim 3-6 \mathrm{~km}$ containing a plausible fraction of ${ }^{26} \mathrm{Al}$ would melt in the centre. A central low-pressure waterdroplet mixture was envisaged, insulated by a surrounding icy shell, the water phase being maintained against refreezing for timescales of $\sim$ Myr.
We now consider an approximate model of a radiogenically heated comet to compute its thermal evolution. Merk \& Prialnik (2003) derive the heat transfer equation

$C_{p} \frac{d T}{d t}=\tau^{-1} X_{0} H e^{\left(-t_{0}+t\right) / \tau}-\frac{3 \kappa T}{R^{2} \rho}$

where $T$ is the average temperature, $C_{p}$ is the average specific heat, $H$ is the radioactive heat input per unit mass, $\kappa$ is the average thermal conductivity, $X_{0}$ is the mass fraction of ${ }^{26} \mathrm{Al}$ and $R$ is the cometary radius. Here $t_{0}$ (assumed to be $1 \mathrm{Myr}$ ) is the time between injection of ${ }^{26} \mathrm{Al}$ and the formation of comets, and $t$ is the time after the comet was formed.

We assume $C_{p}=10^{3} \mathrm{~J} \mathrm{~kg}^{-1}, \rho=0.3 \mathrm{~g} \mathrm{~cm}^{-3}, \alpha=\kappa /\left(\rho C_{p}\right)=$ $10^{-6} \mathrm{~m}^{2} \mathrm{~s}^{-1}$, for porous cometary material, and the previously assumed values of $X_{0}$ and $H$ relating to ${ }^{26} \mathrm{Al}$. (The Merk-Prialnik equation does not lend itself immediately to decay energies from two distinct nuclides, so ${ }^{60} \mathrm{Fe}$ decays are not covered. The inclusion of ${ }^{60} \mathrm{Fe}$, to be taken account of in a subsequent paper, enhances comet melting.) Any transport of energy through convection would be stalled once percolating water vapour recondensed and effectively sealed pores in the frozen body of the comet.

Figure 1 shows the computed temperature by numerically solving Eqn (7) with $T_{0}=60,100 \mathrm{~K}$. For $T_{0}=100 \mathrm{~K}$, comets with radii $R>10 \mathrm{~km}$ reach $273 \mathrm{~K}$, and for $T_{0}=60 \mathrm{~K}$ comets with radii $R>15 \mathrm{~km}$ do likewise at times $t=t_{1}<0.5 \mathrm{Myr}$. The subsequent thermal evolution $T>273 \mathrm{~K}, t>t_{1}$ as calculated according to Eqn (3) is of course unrealistic. Once the temperature $T_{m}=273 \mathrm{~K}$ is reached, further heat released from radioactive decay is absorbed by the melting ice and $T$ does not continue to rise. The energy balance equation at later times is given by

$H_{m} \frac{d Y}{d t}=X_{1} H \tau^{-1} e^{-t / \tau}-\frac{3 \kappa T_{m}}{\rho R^{2}}$

where $Y$ refers to the volume fraction of the comet melted at time $t$ and $X_{1}$ is the remaining fraction of ${ }^{26} \mathrm{Al}$ when the melting temperature is first reached

$X_{1}=X_{0} \exp \left(-t_{1} / \tau\right)$

The above equations due to Jewitt et al. (2007) lead to the solution

$Y(t)=H_{m}^{-1}\left[X_{1} H\left(1-e^{-t / \tau}\right)-\frac{3 \kappa T_{m}}{\rho R^{2}} t\right]$

which is easily evaluated for specified values of the parameters. Figure 2 shows the volume fractions calculated for the two cases $R=12 \mathrm{~km}$ and $15 \mathrm{~km}$.

It is seen here that for smaller comets, even when they have radiogenically produced liquid cores, their volume fractions and durations are less than for larger comets. However, for a plausible set of parameters we see that primordial water in comets is possible even for comets as small as $12 \mathrm{~km}$. These results are generally in agreement with the trends shown in 

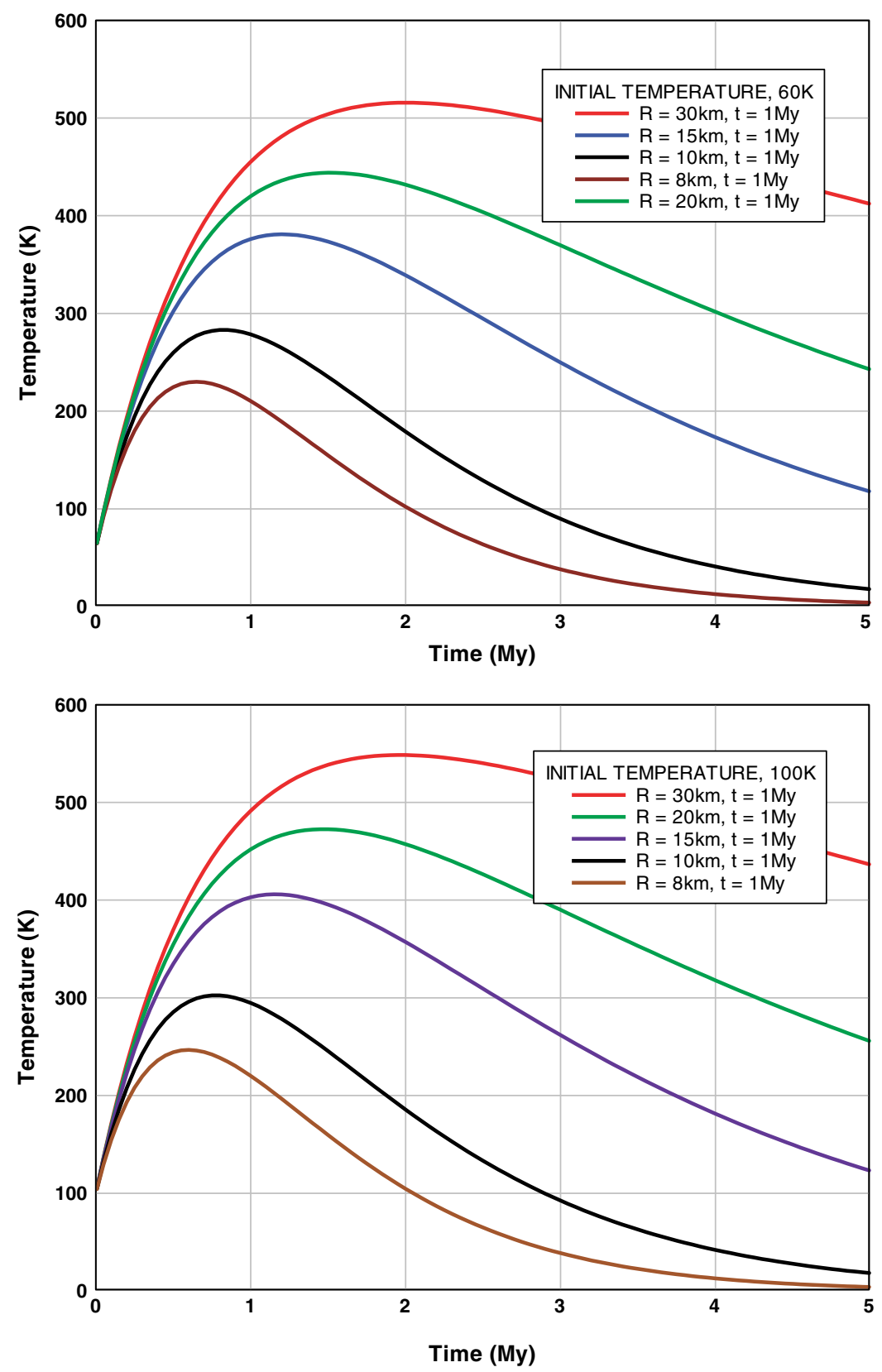

Fig. 1. Calculation of the average internal temperature of a comet accumulated $1 \mathrm{Myr}$ after incorporation of ${ }^{26} \mathrm{Al}$ as a function of the subsequent time. Temperatures above 273 are artifacts of the idealized model. The temperature would flatten and subsequently decline after $273 \mathrm{~K}$ is reached.

calculations by other authors (Jewitt et al. 2007). However, uncertainties of the precise limiting comet size for liquid formation persist and are attributable to uncertainties in relevant thermal constants and/or detailed physical processes involved in the transport of energy through the comet.

The importance of radiogenic heating for exobiology lies in the mixtures of water, minerals and organics in liquid-vapour cometary cores providing an excellent environment for anaerobic microorganisms over Myr timescales. Nutrients are available both from the condensates on interstellar grains and from the mineral grains, to potentially support an ecology based on chemoautotrophs.

\section{Melting under solar heating}

The first space probes to a comet in 1986 explored comet Halley at close range and made discoveries that were at odds with the icy conglomerate model of ice-hydrates mixed with mineral dust. As predicted by Hoyle \& Wickramasinghe (1986) the comet's surface turned out to be exceedingly black, with albedos $\sim 3 \%$, blacker than soot as to be hardly visible. Mass spectroscopy with Giotto instruments revealed a dust component made almost entirely of light elements (called CHON) as well as chondritic and mixed (carbonaceous chondrite) components, in roughly equal quantities. 


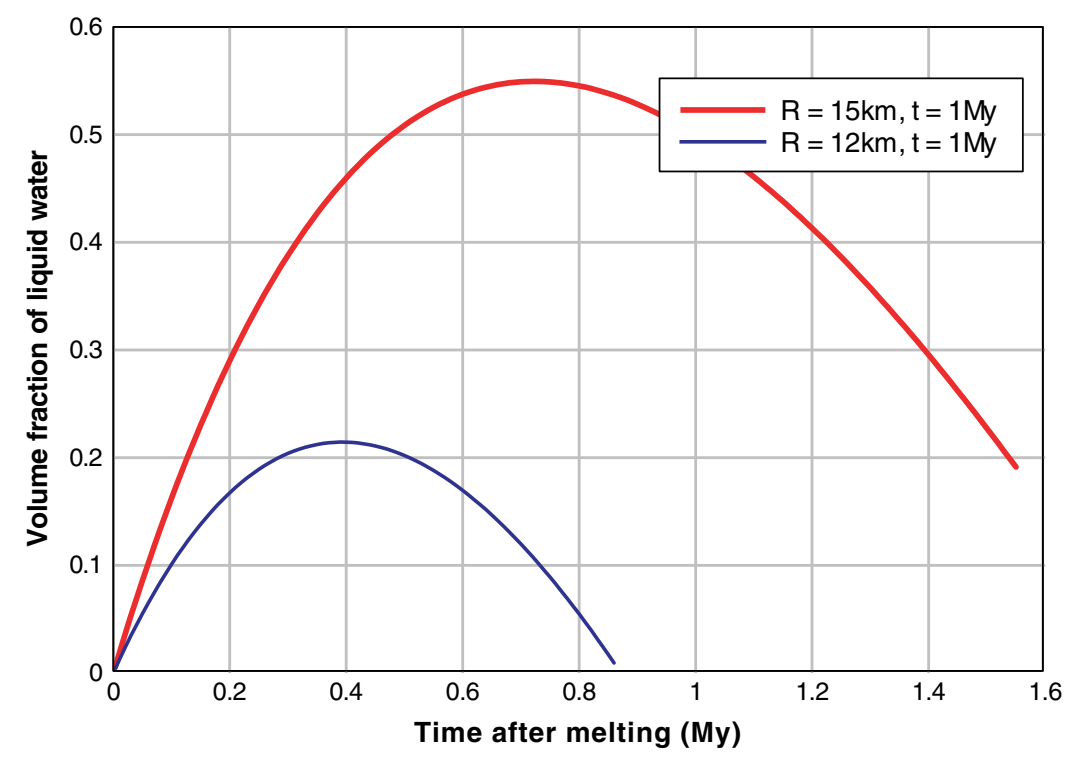

Fig. 2. Volume fractions of water sustainable after melting first occurs.

Infrared maps of comet Halley by Vega 1 showed temperatures in the range $T \approx 320-400 \mathrm{~K}$ at $0.8 \mathrm{AU}$, considerably higher than the sublimation temperature of water ice in space $\sim 200 \mathrm{~K}$. More recent studies of comets have led to similar conclusions on the whole. Comet 19/P Borrelly, observed by Deep Space 1 in 2001 at 1.4 AU, showed a very dark surface with an average albedo $\sim 3 \%$, but with local spots even darker with albedos of less than 0.008 -darker than carbon black. Again, surface temperatures were found to be in the range $300-340 \mathrm{~K}$, with no traces of water. The surface temperatures $300-340 \mathrm{~K}$ at $1.4 \mathrm{AU}$ are appropriate to solar heating with little thermal conduction. Comet 81/P Wild 2, observed by Stardust, also showed similar low albedos, as did comet Tempel 1 (see Interpreting results from Deep Impact, below).

The observation of comets showing the presence of insulating crusts that resist high sub-solar temperatures prompted the model that is now described. Our objective will be to discover whether liquid water can be produced by solar heating at some depth below the surface crust.

We first consider a non-rotating stationary comet under sub-solar insolation with a view to solving the heat conduction equation

$\frac{\partial T}{\partial t}=\kappa \nabla^{2} T$

which in a one-dimensional infinite plane approximation simplifies to

$\frac{\partial T}{\partial t}=\kappa \frac{\partial^{2} T}{\partial z^{2}}$

We envisage a layered one-dimensional structure to emerge over a depth of $\sim 1 \mathrm{~m}$ of comet material due to outgassing near perihelion passage. Over this depth porous organic lag material would accumulate following percolation/sublimation of volatiles, but the outermost $1-2 \mathrm{~cm}$ would inevitably

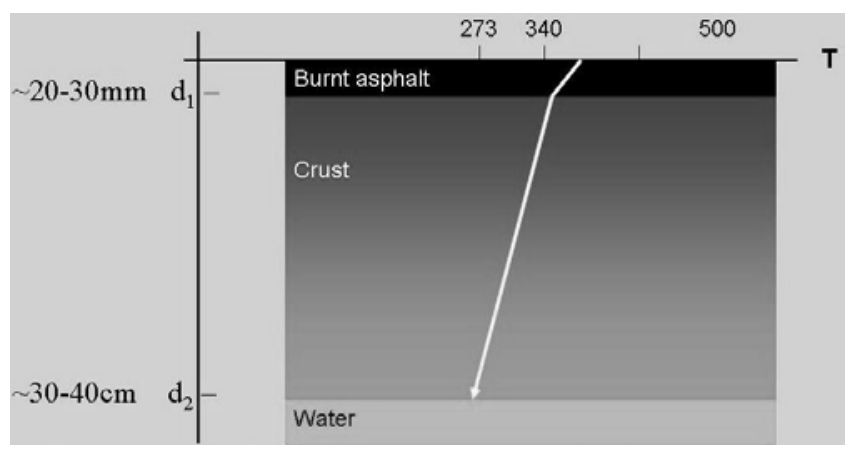

Fig. 3. A schematic temperature profile of a comet.

comprise a sun-burnt insulating crust that can frequently crack and reseal under the pressure $\sim 10 \mathrm{mb}$ of escaping water vapour and volatiles. Organic volatiles reaching the surface would continually reseal and strengthen this 'mobile' outer crust. Below a certain depth of organic material we envisage the possibility of liquid water being maintained through solar heating and with the saturation vapour pressure $\sim 6 \mathrm{mb}$ supported by overlying crust.

Consider a three-layer structure with burnt insulating material at the top from $z=0$ to $z=d_{1}$ with thermal conductivity $\kappa_{2}=0.17 \mathrm{~W} \mathrm{~m}^{-1} \mathrm{~K}^{-1}$ (corresponding to bitumen), a layer with $d_{1}<z<d_{2}$ possessing conductivity $\kappa_{1}=1.7 \mathrm{~W} \mathrm{~m}^{-1} \mathrm{~K}^{-1}$ (organic-ice mix). The possibility of liquid water is considered for a depth $z>d_{2}$.

The energy balance is controlled by the receipt of solar radiation at $r \mathrm{AU}$

$F_{0}=\frac{1.4}{(r / A U)^{2}} k W / m^{2}$

on the comet's surface, and the thermal re-emission

$\sigma \varepsilon_{I R} T^{4}$ 


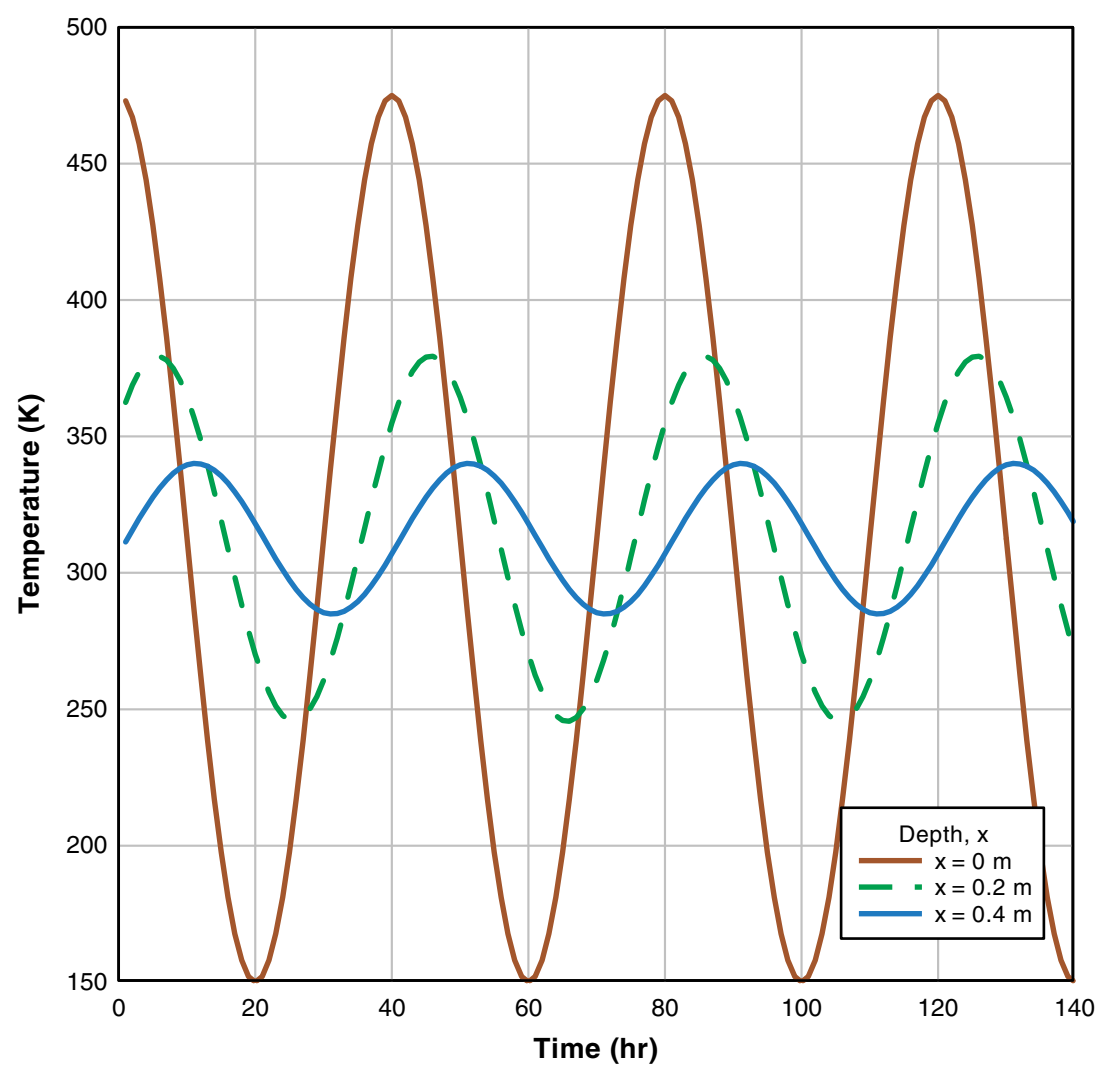

Fig. 4. Variations of temperature with time at depth, $x$ beneath an insulating crust of comet with emissivity $\varepsilon=1$ at 0.7 AU rotating with period 40 hours.

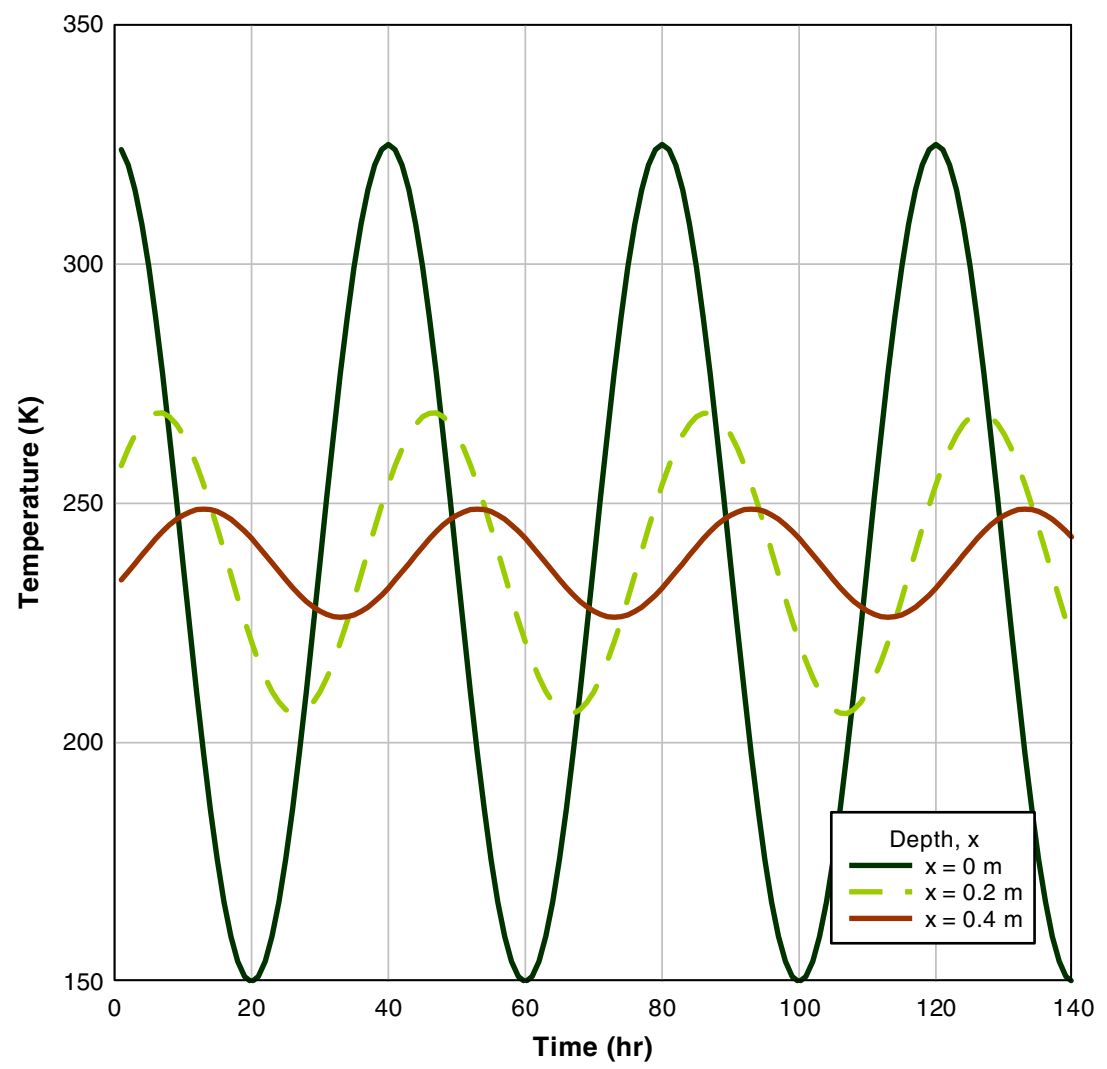

Fig. 5. Variations of temperature with time at depth, $x$ beneath an insulating crust of a comet with emissivity $\varepsilon=1$ at 1.5 AU rotating with period 40 hours. 
which yields

$\sigma \varepsilon_{I R} T^{4}=\frac{(1-A) F_{0}}{(r / A U)^{2}}-\Phi_{d o w n}$

where

$\Phi_{\text {down }}=\kappa \frac{d T}{d z}$

is the net downward flux of radiation during the day and $A$ is the albedo, $A$ being taken to be 0.03 .

If water is mixing and convecting in a steady state of heat flow the solution satisfies

$\Phi_{\text {down }}=\kappa_{1}\left(T_{0}-T_{i}\right) / d_{1}=\kappa_{2}\left(T_{i}-273\right)$

where $T_{0}$ is the maximum outer temperature of the comet, $T_{i}$ is the temperature between the outermost burnt layer and the organic crust, and $273 \mathrm{~K}$ is the interface temperature between the organic crust and the water layer.

The outer temperature $T_{0}$ is calculated from Eqns (11) and (13), and a schematic temperature resulting from such a calculation is set out in Fig. 3.

We now consider a hypothetical layered structure as described above, but with the outer surface exposed to diurnal variations as it would be for a tumbling comet. In the case of comet Halley the tumbling period is $90 \mathrm{~h}$. For a comet rotating with period $\tau$, the temperature at a depth $x$ below an area of thin insulating crust is approximated by a harmonic function. We use an infinite half-plane model to solve the heat conduction equation

$\frac{\partial T}{\partial x}=\alpha \frac{\partial^{2} T}{\partial x^{2}}$

where $\alpha=\kappa / C \rho, \kappa$ being the thermal conductivity, $C$ the thermal capacity and $\rho$ the density. With values appropriate to the organic comet material $C=0.8 \mathrm{~J} \mathrm{~cm}^{-3} \mathrm{~K}^{-1}$, $\kappa=1.7 \mathrm{~W} \mathrm{~m}^{-1} \mathrm{~K}^{-1}$ we have $\alpha=0.003 \mathrm{~m}^{2} \mathrm{~h}^{-1}$ for use in (7) and a thermal skin depth $\sim 30 \mathrm{~cm}$.

For an infinite half-plane maintained at the surface $x=0$ with an oscillatory (sine-wave) temperature variation of amplitude $T_{\text {ampl }}$ and period $\tau$ about a mean temperature $T_{\text {mean }}$ (determined by long-term solar insolation) the analytical solution of (7) is

$$
\begin{aligned}
T(x, t)= & T_{\text {mean }}+T_{\text {ampl }} \cos \left(\sqrt{\frac{\pi}{\alpha \tau}}-2 \pi \frac{t}{\tau}\right) e^{-\sqrt{\frac{\pi}{\alpha \cdot} x^{2}}} \\
& +\frac{2 T_{\text {initial }}}{\sqrt{\pi}} \int_{0}^{x / 2 \sqrt{\alpha . t}} e^{-\xi^{2}} d \xi
\end{aligned}
$$

where $T_{\text {initial }}$ is the constant initial temperature of the medium (Carslaw 1921).

We solve (15) numerically with $\alpha=0.003 \mathrm{~m}^{2} \mathrm{~h}^{-1}$ as an appropriate value for the organic matrix underneath the burnt crust. Figures 4 and 5 show the variations of temperature with time at various depths below the insulating crust. Figure 4 is for a comet at $0.7 \mathrm{AU}$ and Fig. 5 is one at $1.5 \mathrm{AU}$, where the maximum subsolar temperatures are determined by midday solar heating assuming an emissivity $\varepsilon=1$.

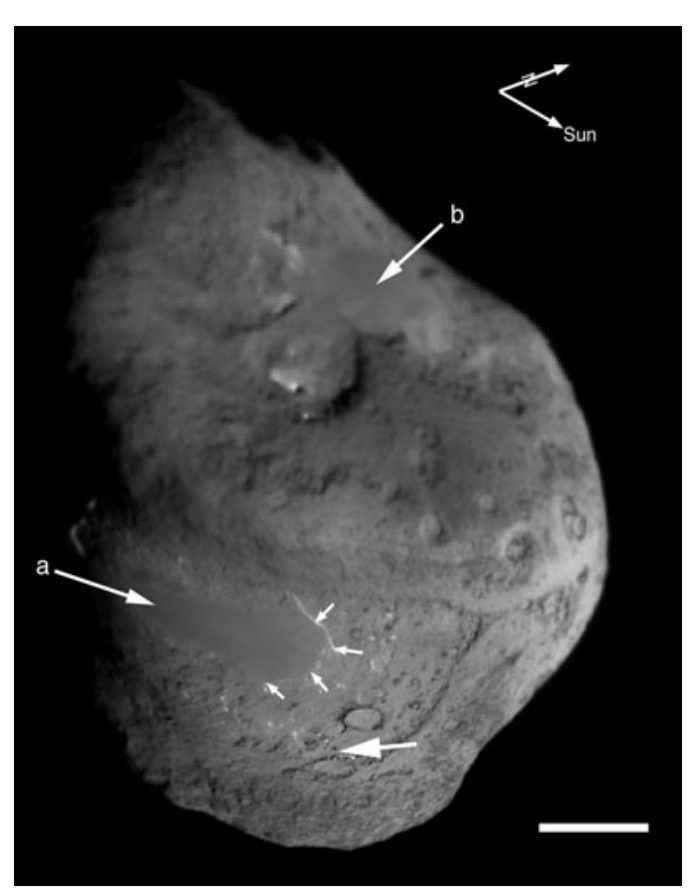

Fig. 6. High-resolution image of Tempel 1.

In Fig. 4 we note that liquid water can persist below $0.2 \mathrm{~m}$ (e.g. comet Halley); in Fig. 5 (e.g. comet Tempel 1) no liquid water is possible. According to these calculations the generation of transient subsurface liquid water domains requires the temperatures at some depth to remain above a notional melting temperature ( $T=273 \mathrm{~K}$ assumed, but could be lower, even $250 \mathrm{~K}$ for organic-water-ice mixtures) throughout a diurnal cycle. For perihelion distances less than $1 \mathrm{AU}$ this could be feasible at depths $\sim 0.3-0.5 \mathrm{~m}$, subsurface pools persisting for days or weeks near perihelion.

\section{Interpreting results from Deep Impact}

The composition and structure of the crust and subcrustal layers of a comet came sharply into focus on 4 July 2005. NASA's Deep Impact Mission dispatched a $370 \mathrm{~kg}$ projectile at a speed of $\sim 10 \mathrm{~km} \mathrm{~s}^{-1}$ to crash head-on into comet Tempel 1. Large quantities of gas and grains were expelled to form an extended plume and coma. The grains were largely micron-sized particles, implying the target was composed of low density friable material (A'Hearn et al. 2005).

The images showed two areas of smooth terrain that we interpret as refrozen lakes (Fig. 6). The southern one curves round the perimeter and shows a sharp boundary on the sunward margin in the form of a $30 \mathrm{~m}$-high scarp. The two circular 'craters' sunward of the scarp have elevated mesas surrounded by 'moats'. These are interpreted as ice impact melts that have developed stable attached crusts helping them resist sublimation, while the surrounding surface has sublimated away over many orbital periods. The smallest craters seen on Tempel 1 also have central peaks that could not be produced by impacts into snow-like material. These are likewise interpreted as impact melts that develop consolidated 

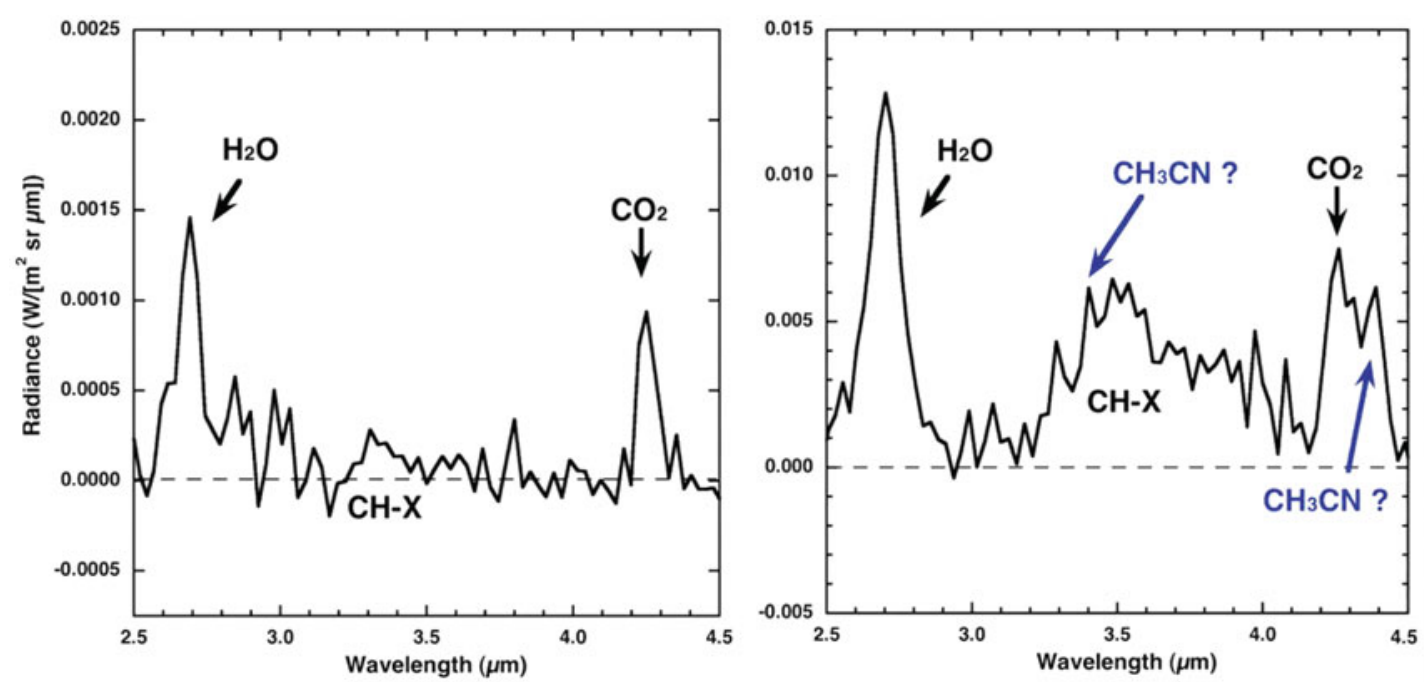

Fig. 7. Spectra of coma of Tempel 1. Ten minutes before (left panel) and four minutes after the impact (right panel) on 4 July 2005.

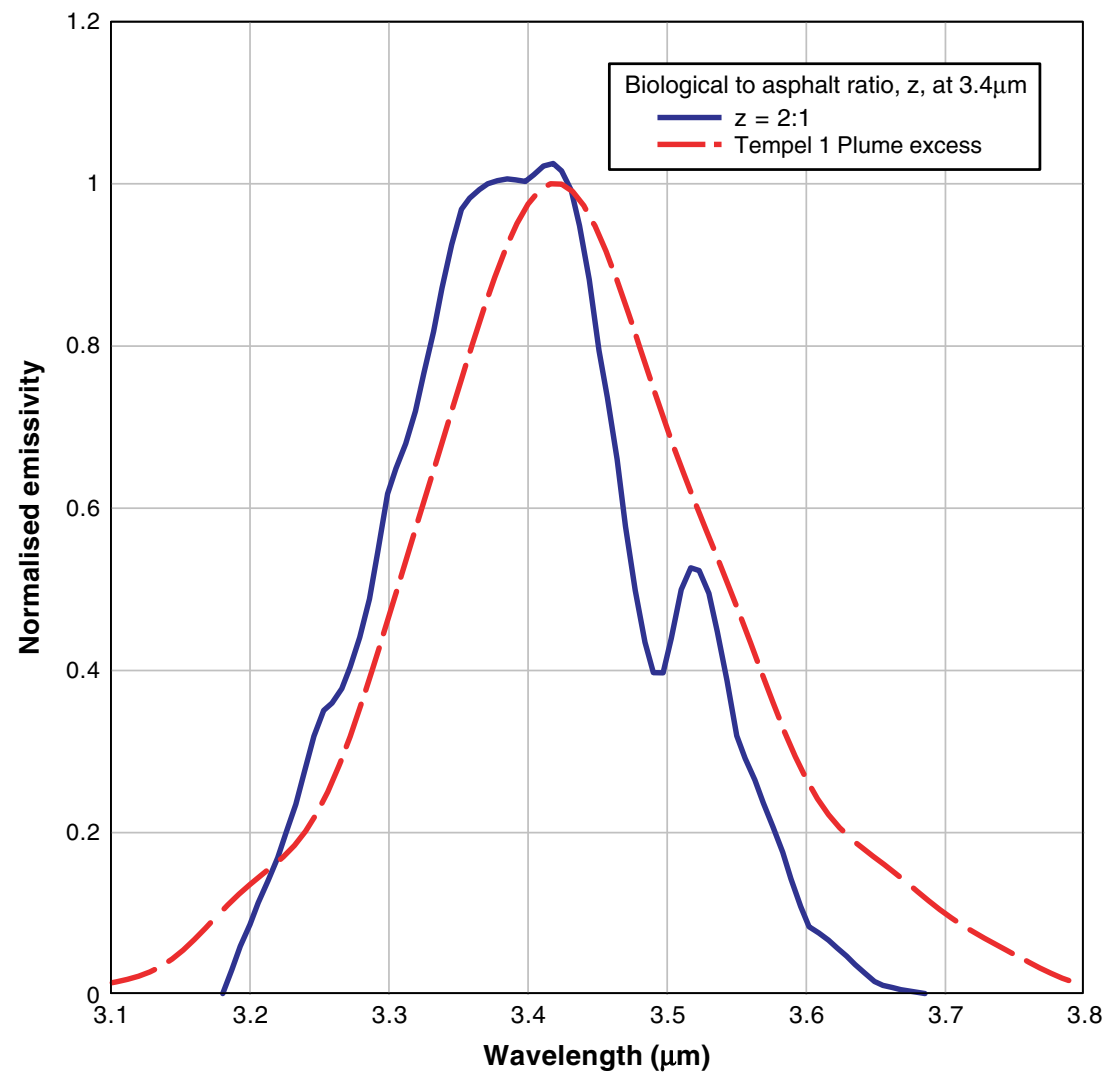

Fig. 8. Normalized opacities near $3.4 \mu \mathrm{m}$ for a mix of 'normal' cometary dust matching biological material with asphalt. For comparison is plotted the 'unexplained' component of emission from the plume following impact on 4 July 2005.

stable crusts, while the surrounding material erodes more quickly.

Spectral studies identify chemical components of the impact-generated plume of gas and grains and led in particular to the new discovery of a clay feature (Lisse et al. 2005). Spectra of the coma in the near infrared waveband $10 \mathrm{~min}$ before and 4 min after the impact obtained by A'Hearn $e t$ al. (2005) show the impact caused a 10 -fold increase in $\mathrm{H}_{2} \mathrm{O}$ and
$\mathrm{CO}_{2}$ and, most marked, a strong relative increase of $\mathrm{CH}$ emissions over the waveband 3.3-3.5 $\mu \mathrm{m}$ (Fig. 7). The relative surge in $\mathrm{CH}$ indicates these organic volatiles present in micron-particle 'snow' do not normally emerge in the cometary gases, but are filtered by the surface crust, helping seal it, as presumed in the above section, Melting under solar heating. Likewise, the clay particles (below) may also be retained in a normally gassing comet. 


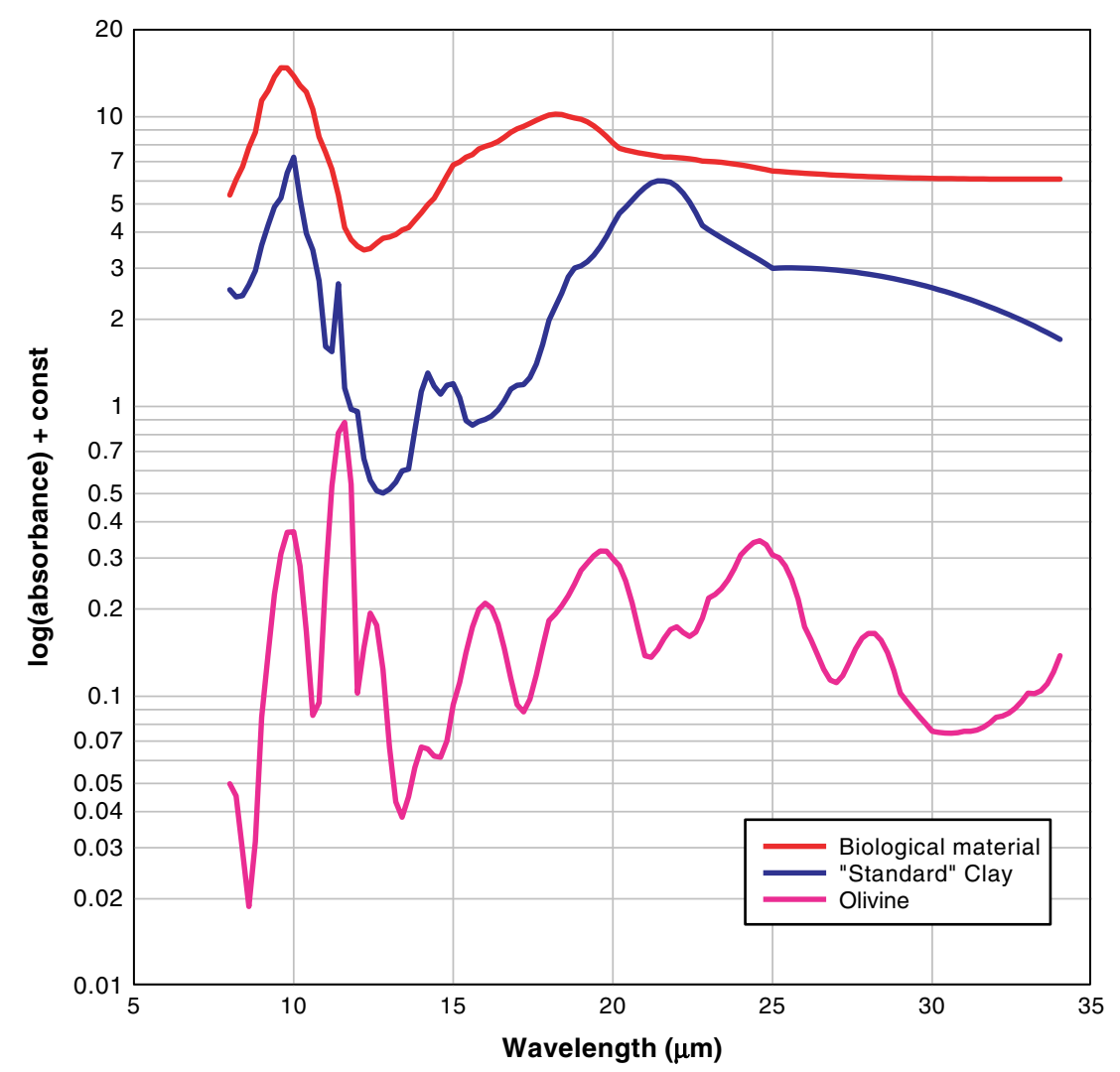

Fig. 9. Absorbance data used for the calculations - for olivine and biomaterial the data are taken from compilations by Wickramasinghe \& Hoyle (1999).

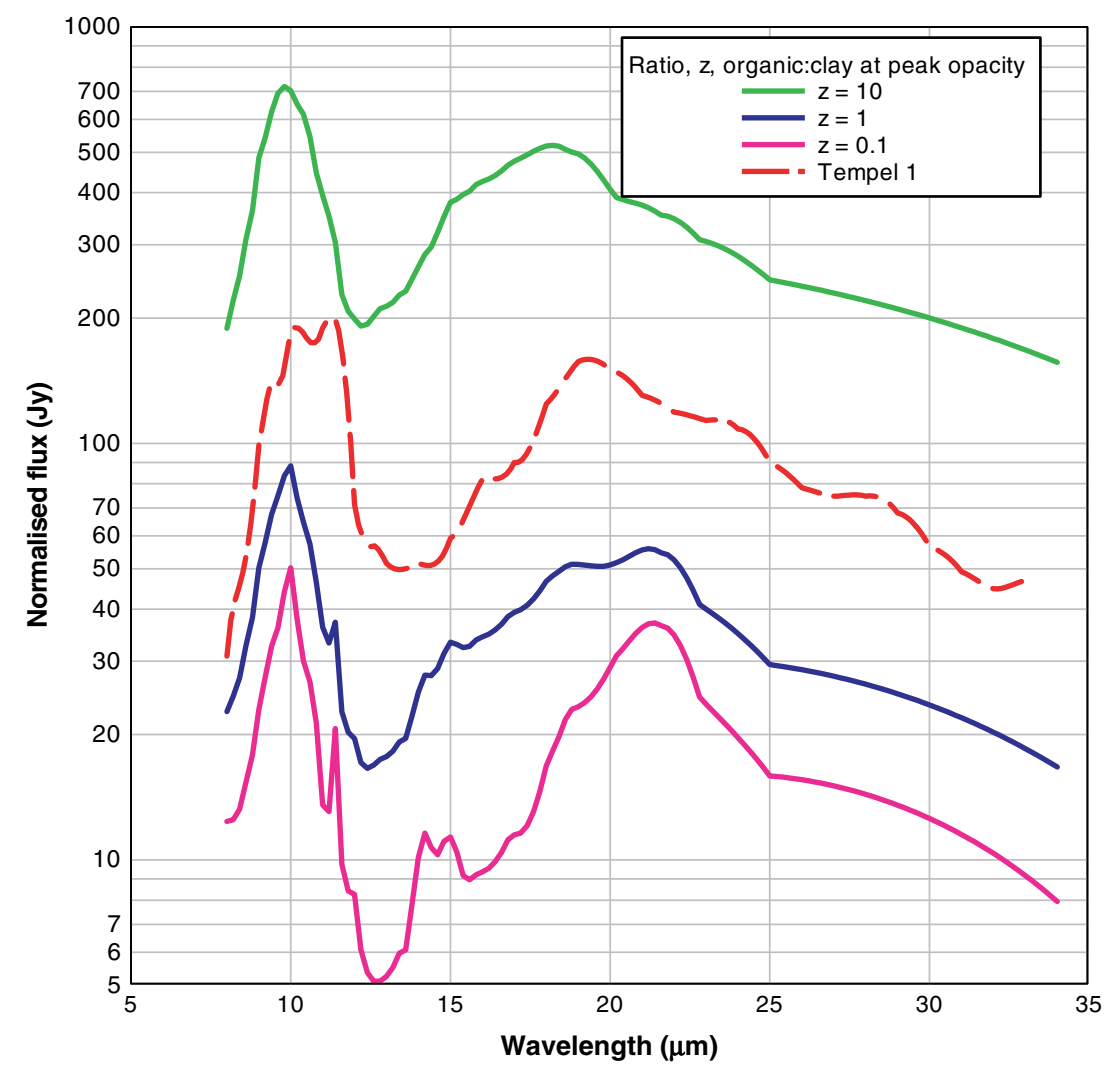

Fig. 10. Intensity of radiation (Jy) due to mixtures of biologic material and clay. $z$ is the ratio of opacity contributions (biologic: olivine) near the $9 \mu \mathrm{m}$ peaks. The dashed curve represents data for Tempel 1. 


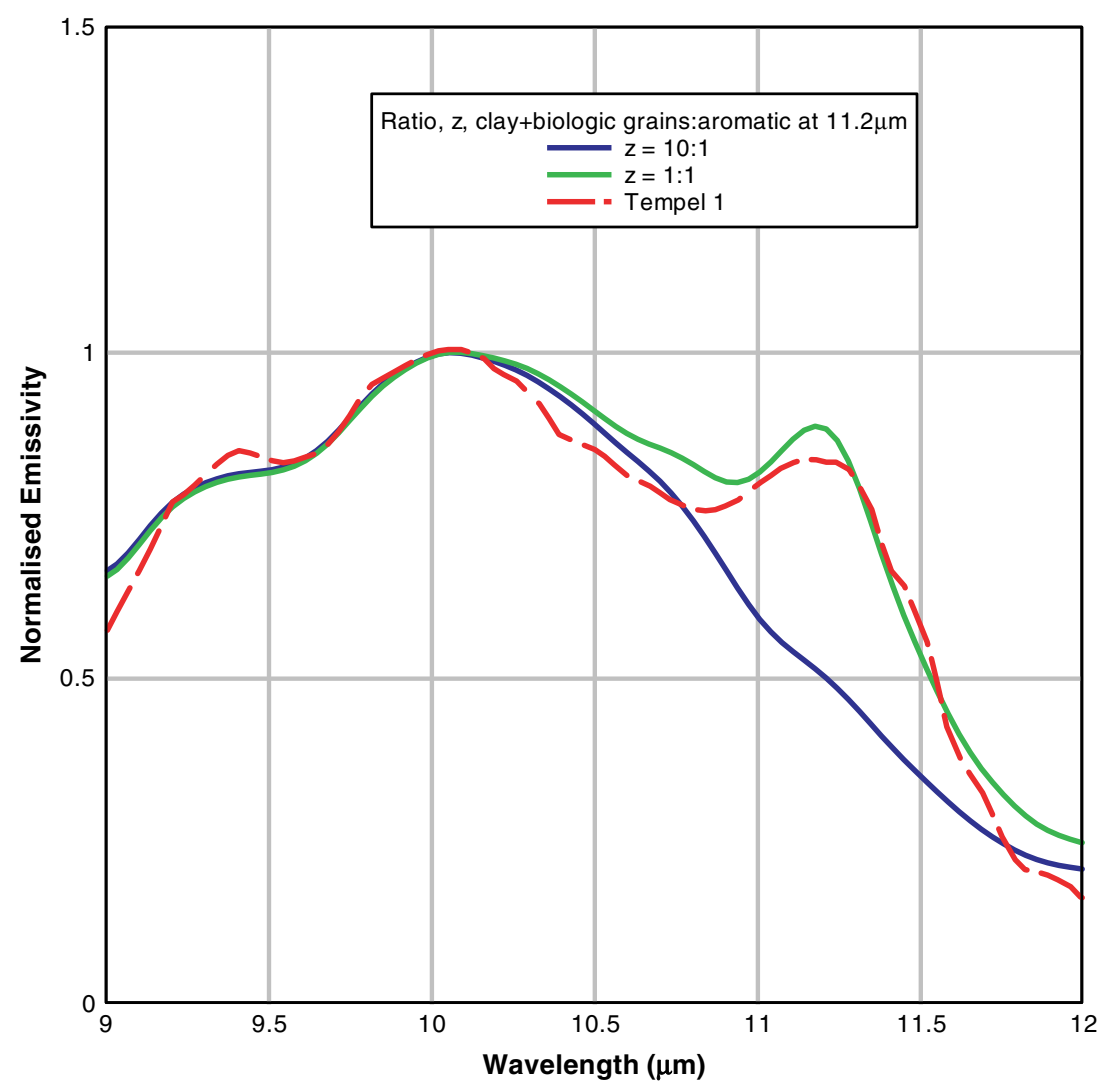

Fig. 11. Normalized opacities near $10 \mu \mathrm{m}$ due to mixtures of clays and biological aromatic molecules. Comparable contributions at $11.2 \mu \mathrm{m}$ from clays and an ensemble of aromatic molecules (half width at maximum intensity of $\sim 0.3 \mu \mathrm{m}$ ) fit most of the long-wave opacity requirements.

The excess radiation over this waveband in the post-impact plume (apart from features in inorganic coma gases) is best modelled on the basis of degraded biologic-type organic material. We consider the type of organic dust discussed by Hoyle \& Wickramasinghe (1991) for explaining the mean 3.3-3.5 $\mu \mathrm{m}$ emission profile for comet Halley and other comets (organic/biologic). We also include asphaltic material presumed to result from its degradation, which is used as an analogue of refractory organic solids on primitive solar system bodies. Space radiation and solar heating are seen as degrading organic-based asphaltite (Vernazza et al. 2005).

Figure 8 shows the normalized opacities calculated for such a model compared with the observations of the post-impact plume of Tempel 1. We see that with a 2:1 contribution of biological material to asphalt in the opacity at $3.4 \mu \mathrm{m}$ an approximate match with the data is obtained (note that the resolution of the data is relatively low). The match is satisfactory considering that asphalt is likely to be an oversimplified representation of degraded biomaterial (Vernazza et al. 2005).

Observations of Tempel 1 some 20 hours following impact using the Spitzer Telescope yielded 5-37 $\mu \mathrm{m}$ spectra generally similar to the spectra of Comet Hale-Bopp taken by Infrared Space Observatory. The Hale-Bopp data was interpreted by Wickramasinghe \& Hoyle (1999) on the basis of a model involving olivine and biologic-type material in the mass ratio
1:10. To model the Tempel 1 data we considered mixtures of olivine and biomaterial with normalized absorbance curves as given in Fig. 9.

The two materials were conceptually mixed with opacity contributions at their peak maxima in the ratio organic: olivine $=z: 1$. Since for olivine/clay the maximum mass absorption coefficient near the $9 \mu \mathrm{m}$ peak is over five times that of the less well-ordered organics, the corresponding mass ratios are near $5 z: 1$.

Figure 10 displays computed flux curves for mixtures of clay and biologic grains heated to $350 \mathrm{~K}$. The mass ratios of organics to clay are again well in excess of $10: 1$. The temperature $300-350 \mathrm{~K}$ matches the underlying continuum of the Tempel 1 dust spectrum.

The spectra of clays tend to be somewhat variable one to another, reflecting their idiosyncratic mineral configurations. However, fine structure in the $8-11 \mu \mathrm{m}$ absorption band centred near 9, 10 and $11 \mu \mathrm{m}$ can in general be regarded as signifying a clay, and these indeed have been found in the higher resolution spectra of Tempel 1 following the Deep Impact event.

Over the $8-13 \mu \mathrm{m}$ wavelength interval we combined the standard clay spectrum given by Frost et al. (2000) with three other clay spectra, yielding the normalized emissivity plotted in Fig. 11. The average emissivity spectrum (post-impact) of Tempel 1, shows good agreement with clay near 9 and $10 \mu \mathrm{m}$, 
but a significant excess is centred near $11.2 \mu \mathrm{m}$ (Lisse et al. 2005). This 'missing' flux has been attributed to polycyclic aromatic hydrocarbons (PAHs) although individual compact PAHs have too narrow $11.2 \mu \mathrm{m}$ features. We use instead the ensemble of biological aromatic molecules that model the interstellar 'unidentified infrared bands' (Hoyle \& Wickramasinghe 1991). Figure 11 shows that this results in satisfactory overall agreement with the observations.

\section{Concluding remarks}

We confirm the conclusions of Lisse et al. (2005) that clay minerals, complex organics and PAH-type material are present in the post-impact ejecta from Tempel 1. Earlier claims in the last decade of similar materials in interstellar dust particles and in $\mathrm{C} 1$ carbonaceous chondrites cannot now be discounted. Since clay minerals are thought to form only in contact with liquid water, the target area on Tempel 1 would have included refrozen subcrust and not simply pristine condensates from the pre-solar nebula.

That the solar nebula condensation was triggered by a supernova is favoured by modern evidence of extinct shortlived radionuclides. Comets that formed in $\sim 1 \mathrm{Myr}$ would have developed radioactively heated liquid cores, in which anaerobic microorganisms deriving from the pre-solar nebula could have evolved in isolation for several Myr. As the radiogenic heating decayed, the ecosystem would become deep frozen and dormant in a low-radiation environment. Only after the outer comet layers sublimate away could the microorganisms be released for onwards dispersal, perhaps after a relatively brief period in the warmed sub-crustal stage.

Transient sub-surface pools with organic nutrients and viable microbes allow biological activity to restart seasonally at the periodic returns of comets. Hoover et al. (2004) have suggested on the basis of Antarctic flora that a wide range of species which can withstand periodic deep freezing could potentially flourish. The pools in our model are maintained a few tens of centimetres below a sealed outer crust that is continually regenerated due to condensation of diffusing organic materials. The crust can readily withstand the $6 \mathrm{mb}$ pressure needed to support a water interface, but sporadic rupturing of areas of crust would occur, leading to episodes of outgassing. Such rupturing could arise from impacts on the surface, or gases building up pressure.

The liquid pools themselves would be maintained by the tensile strength of the overlying layer which could be 10-100 times the $6 \mathrm{mb}$ water vapour pressure. If, however, gas pressure builds up due to metabolic activity in sub-surface niches, the tensile strength would be insufficient to maintain equilibrium and the crust will be ruptured, leading to the release of gas and dust (Wickramasinghe et al. 1996).

Microorganisms do not need a body of liquid water for metabolism and replication. A naturally occurring population of bacteria in Siberian permafrost is known to have a doubling time ranging from 20 days at $-10{ }^{\circ} \mathrm{C}$ to 160 days at $-20{ }^{\circ} \mathrm{C}$ (Rivkina et al. 2000). But we conceive that liquids enhance metabolism strongly, due to the transport of nutrients and wastes, more than the metabolic processes per se. Transient subsurface pools in comets thus provide ample scope for amplification and dispersal of dormant microorganisms.

\section{References}

A'Hearn, M.F. et al. (2005). Science 310, 258.

Brownlee, D.E. (1978). In Protostars and Planets, ed. Gehrels, T., p. 134. University of Arizona Press, Tuscon.

Carslaw, H.S. (1921). The Conduction of Heat. Macmillan and Co, London. Diehl, R. et al. (1997). In AIP Conference Proceedings, eds Dermer, C.D. et al., vol. 410, p. 1114. American Institute of Physics.

Frost, R.L., Ruan, H. \& Kloprogge, J.T. (2000). Int. J. Vib. Spectros. 4 (1), $1-12$.

Hoover, R.B., Pikuta, E.V., Wickramasinghe, N.C., Wallis, M.K. \& Sheldon, R.B. (2004). Astrobiology of Comets. In Instruments, Methods, and Missions for Astrobiology VIII (Proceedings of SPIE, 5163), pp. 191-202. SPIE (www.astrobiology.cf.ac.uk/SPIE2004.pdf).

Hoover, R.B., (2005). In Perspectives in Astrobiology, eds Hoover, R.B., Rozanov, A.Y. \& Paepe, R.R., vol. 366, p. 43. IOS Press, Amsterdam.

Hoyle, F. \& Wickramasinghe, N.C. (1979). Diseases from Space, J.M. Dent and Sons, London.

Hoyle, F. \& Wickramasinghe, N.C. (1986). Earth Moon Planets 36, 289.

Hoyle, F. \& Wickramasinghe, N.C. (1991). The Theory of Cosmic Grains. Kluwer Academic Press, Dordrecht.

Jewitt, D.C., Chizmadia, L., Grimm, R. \& Prialnik, D. (2007). In Protostars and Planets, eds Reipurth, V.B., Jewitt, D. \& Keil, K. p. 863. University of Arizona Press, Tuscon.

Lisse, C.M. et al. (2005). Space Science Rev. 117, 161.

MacPherson, G.J., Davis, A.M. \& Zinner, E.K. (1995). Meteoritics 30, 365.

McSween, H.Y. (1979). Geochim. Cosmochim. Acta 43, 1761.

Merk, R. \& Prialnik, D. (2003). Earth Moon Planets 92, 359

Mostefaoui, S., Lugmair, G.W., Hoppe, P. \& Goresy, A. El. (2004). New Astron. Rev. 48, 155.

Rivkina, E.M., Friedmann, E.I., McKay, C.P. \& Gilichinsky, D.A. (2000). App. Environ. Microbiol. 66(8), 3230.

Vernazza, P., Mothé-Diniz, T., Barucci, M.A., Birlan, M., Carvano, J.M., Strazzulla, G., Fulchignoni, M. \& Migliorini, A. (2005). Astron. Astrophys. 436, 1113-1121.

Wallis, M.K. (1980). Nature 284, 431.

Whipple, F.L. \& Stefanik, R.P. (1966). Mém. Soc. R. Sci. Liège Sér. 5, 12, 33.

Wickramasinghe, N.C. \& Hoyle, F. (1999). Astrophys. Space Sci. 268, 379. Wickramasinghe, N.C., Hoyle, F. \& Lloyd, D. (1996). Astrophys. Space Sci. 240, 161.

Yabushita, S. (1993). Mon. Not. Roy. Astron. Soc. 260, 819.

Zinner, E. (2003). Science 300, 265-267. 